\title{
Experimental Study on Solar Energy Storage Using Phase Change Materials in Spherical Shell Storage System
}

\author{
PASAM. BHAGYALAKSHMI ${ }^{*}$, K. RAJAN² and K. R. SENTHIL KUMAR ${ }^{3}$ \\ 'St.Peter's University, Tonakela Camp Road, Sankar Nagar, Avadi, Chennai, \\ Tamil Nadu 600 054, India. \\ 2Department of Mechanical Engineering, Dr.M.G.R.Educational and Research \\ Institute, university, Chennai-600095, India. \\ ${ }^{3}$ Department of Mechanical Engineering, R.M.K.Engineering college, Chennai, India. \\ *Corresponding author E-mail: bhagyalv6@gmail.com
}

http://dx.doi.org/10.13005/ojc/340156

(Received: September 16, 2017; Accepted: October 29, 2017)

\section{ABSTRACT}

Storing of available energy for later use is a necessity in the present world, one such method is using Phase Change Materials (PCMs). PCMs need a heat transfer medium to transfer the energy from the source to the PCM. In this present work spherical balls are used to store the PCM and water is used as a heat transfer fluid. Paraffin wax (PW) and Palmitic acid (PA) and (40$60 \%$ PW-PA) eutectic mixture are used as PCMs. The solidification process is carried out in three different ways as cold water, hot water and natural solidification without water. In case of hot water and natural solidification processes the three materials posses the stable reduction. In cold water solidification process base materials have sudden drop in temperature where as the eutectic mixture has gradual change.

Keywords: Palmitic acid, Paraffin wax, Heat transfer fluid (HTF), Heat exchanger, Eutectic mixture.

\section{INTRODUCTION}

The continuous increase in the level of greenhouse emissions and the increase in fuel prices are the main driving forces behind efforts to more effectively utilize various sources of renewable energy. Solar energy is among the most promising alternative sources of energy compared to traditional fossil fuels ${ }^{1}$.Storage of solar energy are an emerging task to the present world. Energy from the sun is vital but storing this energy for off sun shine hours is a difficult thing. Thermal Energy storage (TES) method is one of the ways to store energy for few minutes to few $h^{2}$. Latent heat storage(LHS) system in TES is one of the effective way of storing energy in large quantities due to its

\footnotetext{
This is an Open Access article licensed under a Creative Commons Attribution-NonCommercial-ShareAlike 4.0 International License (https://creativecommons.org/licenses/by-nc-sa/4.0/), which permits unrestricted NonCommercial use, distribution and reproduction in any medium, provided the original work is properly cited.
} 
high energy storage density ${ }^{3-6}$. Phase change materials are used for storing energy in latent heat storage system due to their good thermo physical properties and also reduced storage volumes compare to sensible heat storage systems $\mathrm{s}^{7,8}$. Basically the PCMs cannot absorb or release heat without medium. They require heat transfer fluids and need of a heat exchanger.

Researchers have been conducted and tested with many PCMs in different ways into a TES system. Gil et al., ${ }^{10}$ have chosen hydroquinone as PCM for medium temperature range for storing the energy in a Shell and Tube heat exchanger and tested with and without fins. Experiments were conducted with five different volumetric flow rates and the effectiveness of the storage tank were calculated. The effectiveness results were found to match with literature when used without fins. Schreiber et al., ${ }^{11}$ have investigated on adsorption thermal energy storage systems for the energy storage density and obtained higher efficiencies during charging and discharging for higher temperature ranges. Concluded that the higher storage density by charging at high temperatures for a short period of time and losses can be reduced for long term storage.

Esapour et al., ${ }^{12}$ have conducted tests on a multi-tube heat exchanger with water as HTF and RT35 as the PCM. Comparison test have done with a double pipe. Results show that as the inlet temperature increases, the melting time reduces, whereas when the mass flow rate increases the melting time reduced to some extent. And also increased the number of tubes from 1-4 and enlarged the melt region which leads to $29 \%$ reduction in melting time. Faegh et al., ${ }^{13}$ have done experimental investigation on solar energy storage using PCM. In this test there is no direct contact between the HTF and the PCM. The energy is transferring to $\mathrm{PCM}$ through condenser. The results showed that the yield increased by $86 \%$ as compared to the system without PCM and an increased efficiency of $50 \%$.

Merlin et al. ${ }^{14}$ have done experimental study on thermal storage using PCM associated with various configurations of conductive structures like finned exchanger, graphite powder and Expanded Natural Graphite and improved the overall heat transfer coefficient. the numerical simulations are also compared to the experimental results and used to investigate the impact of the thermal contact resistance on the heat transfer process of the LTHS system. Medrano et al., ${ }^{15}$ have done investigation experimentally the heat transfer process during melting (charge) and solidification(discharge) of five small heat exchangers working as latent heat thermal storage systems. Commercial paraffin RT35 is used as PCM. Results show that the double pipe heat exchanger with the PCM embedded in a graphite matrix is the one with higher values and the compact heat exchanger is the one with the highest average thermal power.

Hosseini et al., ${ }^{16}$ have investigated numerically and experimentally the effect of fins height and stefan number on the performance of the shell and tube heat exchanger which contains a PCM. The results shown that the increasing stefan number the melting time reduces and the increasing fins' height influences the solidification time than melting time. Mehrali et al., ${ }^{17}$ have prepared a novel form-stable PCM by impregnation method. Palmitic acid is added to graphene nanoplatelets (GNPs) with three different specific surface areas as supporting material. The results showed that the thermal conductivity of the composite was 10 times that of the palmitic acid and the latent heat of the composite is close to the value of pure palmitic acid.

Regin et al., ${ }^{18}$ have conducted a numerical analysis of thermal storage system where Paraffin as a Phase change material. They concluded that the solidification time is too long due to low heat transfer coefficient, phase transition temperatures reduces the melting time and charging and discharging are higher for small radius capsules than larger capsules. Jesumathy et al., ${ }^{19}$ designed an energy storage system to study the heat transfer characteristics of paraffin wax during melting and solidification processes in a vertical annulus energy storage system. The experimental results proved that the PCM melts and solidifies congruently, and the melting front moved from the top to the bottom of the PCM container whereas the solidification front moved from bottom to the top along the axial distances in the PCM container. 
Many researchers have concentrated on paraffin wax and Palmitic acid, tested in different heat exchangers. In this present work paraffin and Palmitic acid eutectic mixture chosen and tested in spherical storage unit ${ }^{20}$. The selection of the materials is based on the literature and availability, to store the solar heated water for a longer period of time even in the bad weather conditions. The results were compared with the base materials results. This eutectic mixture also tested in cylindrical storage unit which is discussed in another paper by the same authors.

\section{Selection of materials and experimental methods}

Paraffin wax is one of the best suited PCM for low temperature applications. Paraffin is cheap and has moderate energy storage density ${ }^{21}$. Palmitic acid is also an organic but non-paraffin group material suited for low temperature applications ranging from 16 to $65^{\circ} \mathrm{C}^{9}$. It has better latent heat capacity and sharp melting point. Both materials have certain good and negative points. To overcome the problems in both, eutectic mixture of the two has chosen. Paraffin wax from organic group and Palmitic acid from fatty acid organic group have mixed in different proportions and DSC test has been conducted. From the DSC test results
$40 \% \mathrm{PW}$ and $60 \%$ PA eutectic mixture has chosen as a better composite for thermal energy storage. Properties have shown in Table. 1.

\section{EXPERIMENTAL}

\section{Experimental Set Up}

Figure. 1 shows the Solar based water heater with a Thermal Energy Storage unit connected to it. The entire unit consists of three main parts. The solar collector, a well insulated water tank and a thermal energy storage tank. The PCM is incorporated in spherical balls and these balls along with its stand placed in the thermal storage tank. The stand is to hold the spherical balls, also serves the purpose of fins. During the sunny hours the water from the overhead tank will be heated and sent to the solar tank. Later it will be sent to the thermal storage tank, where the PCM get charged. The heating continues till the dusk. During the discharging process hot water from the thermal storage unit will be utilized first, later from the solar tank; water will always pass through thermal storage unit to the utility. The entire unit has kept in the terrace and the night temperatures were come down to $28^{\circ} \mathrm{C}$ with a constant breeze in the atmospheric air.

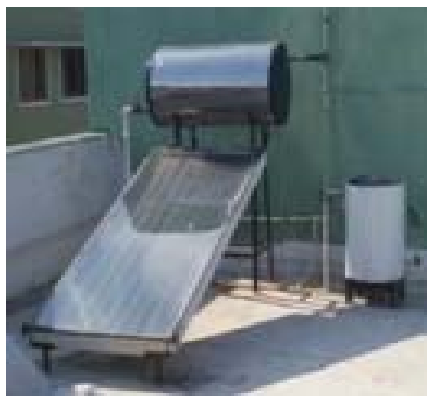

Fig.1. Experimental Set up

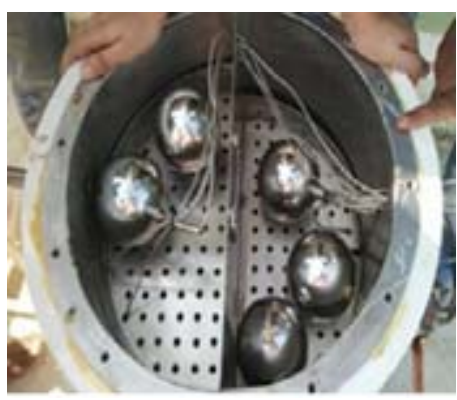

Fig. 2(a) Arrangements of spherical balls in the storage tank

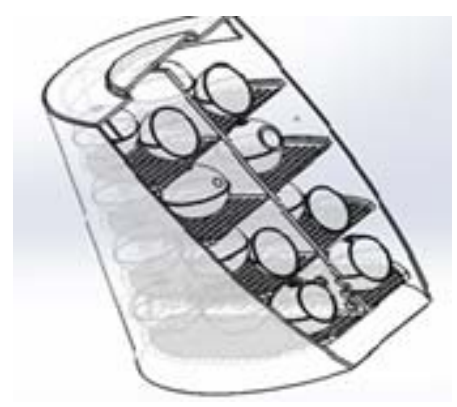

(b) CAD Modeling of storage tank

Table.1: Properties of the Phase Change Materials

\begin{tabular}{lcccc}
\hline Material & $\begin{array}{c}\text { Melting } \\
\text { temp }\left({ }^{\circ} \mathrm{C}\right)\end{array}$ & $\begin{array}{c}\text { Solidification } \\
\text { temp }\left({ }^{\circ} \mathrm{C}\right)\end{array}$ & LHS[charging] $(\mathrm{J} / \mathrm{g})$ & LHS[discharging] $(\mathrm{J} / \mathrm{g})$ \\
\hline Paraffin wax & $58.8-67.2$ & $61.7-52.8$ & 138 & 135.2 \\
Palmitic acid & $62.2-67.9$ & $55.1-61.0$ & 193.7 & 193.7 \\
Eutectic mixture & $52.6-59.8$ & $40.9-47.1$ & 214.7 & 194.6 \\
\hline
\end{tabular}




\section{Thermal Energy Storage Unit with PCM}

The Storage unit consists of a stainless steel cylindrical tank of $40 \mathrm{~cm}$ diameter and $50 \mathrm{~cm}$ height with a detachable lid. It is well insulated from the bottom and peripheral by Foam insulation. The top of the tank contains a steel plate of $0.2 \mathrm{~cm}$ thick circular disc which is bolted to the drum. There is no insulation provided for the top lid. There is an inlet and outlet provision to the HTF. Stainless steel spherical balls of $10 \mathrm{~cm}$ diameter are used for storing the energy. For Spherical balls the arrangements are made inside the tank so that 21 spheres of 100 $\mathrm{mm}$ diameter each are kept in the chamber at 7 different heights as shown in Fig. 2. From each stage thermocouple is provided to measure the temperature.

\section{RESULTS AND DISCUSSIONS}

The experimental tests have been conducted with the eutectic mixture of paraffin and palmitic acid of $40: 60$ ratios and compared with the

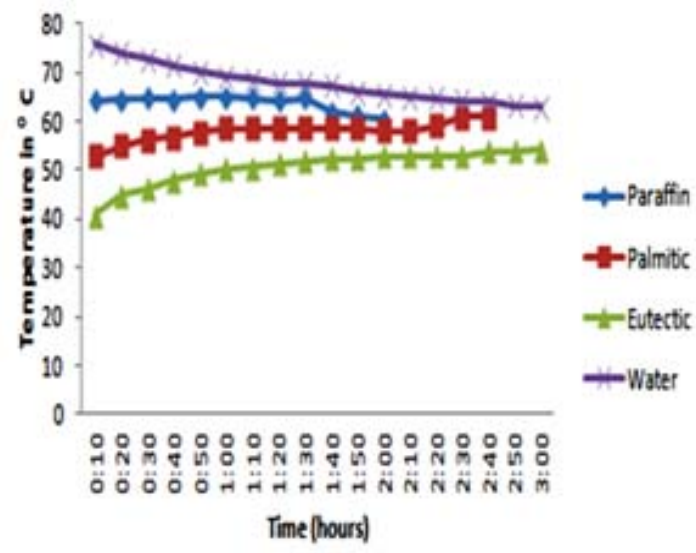

Fig. 3. Melting Curve base materials of paraffin wax and palmitic acids. The time and temperatures have noted for both melting and the three different conditions of solidifications. 1. Solidification with Cold water, 2. Solidification with Hot water and 3.Solidification without HTF. The measured parameters are analyzed and discussed.

\section{Melting process}

The solar heated water filled in the storage tank with an inlet temperature of $75^{\circ} \mathrm{C}$ in all the three cases and for all the three materials. If the temperature is not up to the mark then auxiliary unit is utilized to get $75^{\circ} \mathrm{C}$. Once the storage tank filled with hot water it is considering as starting of the melting process and temperatures have noted for every $10 \mathrm{~min}$. intervals till the temperatures of the material and the HTF are near equal. Each process has repeated for 10 cycles and averaged the values. The melting process remains same in all the cases. The resulting temperatures have plotted in the graph shown in Figure. 3.

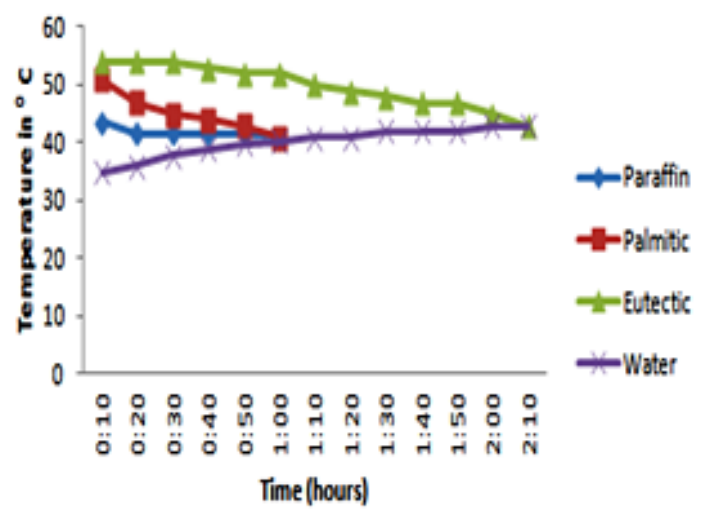

Fig. 4. Cold water solidification curve

Table. 2: Dimensions of the Storage unit

\begin{tabular}{lccc}
\hline Components & Material & Dimensions $(\mathrm{mm})$ & Volume $\left(\mathrm{m}^{3}\right)$ \\
\hline Storage Cylinder & Stainless Steel & $400 \times 500$ & 0.06283 \\
Spherical balls & Stainless Steel & 100 & 0.000524 \\
Insulation & $\quad$ Foam & -1 & $-\overline{1}$ \\
Fins & Stainless Steel & 0.00132 \\
\hline
\end{tabular}




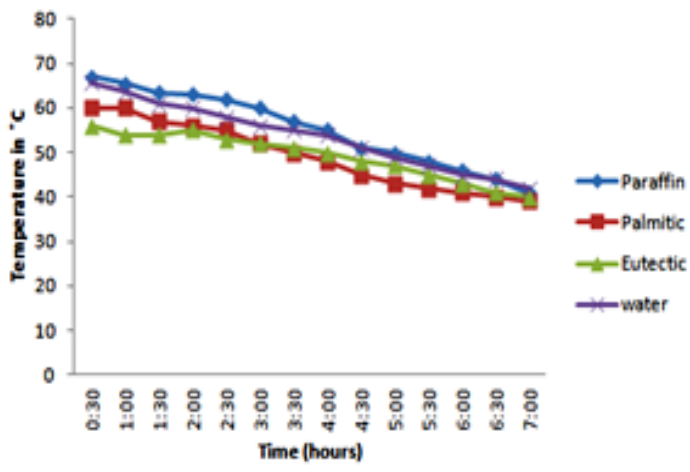

Fig. 5. Hot water solidification curve

\section{Solidification Processes}

Cold water Solidification: In this case once the material attains temperature near the HTF temperature then the hot water is drained and filled the storage tank with cold water. Once the tank is filled with cold water then it is considering as solidification process and recording the temperature variations in both the material and the HTF for every 10 minutes. The pure PCMs have lost their temperatures in less than an hour and the raise temperature of the water was $5^{\circ} \mathrm{C}$ only for this one hour period. The eutectic mixture solidified slowly. The HTF has raised its temperature by $8^{\circ} \mathrm{C}$ in 120 minutes.

Hot water solidification: Solidification process occurred by retaining the hot water in the tank itself. The temperatures have recorded for every 10 min. till both the material and HTF attains $45^{\circ} \mathrm{C}$. The change in temperatures of both material and the HTF for a period of $120 \mathrm{~min}$. it was $5^{\circ} \mathrm{C}$. It took more than $7 \mathrm{~h}$ to come to $45^{\circ} \mathrm{C}$.

Natural Solidification: In this case it has been tested for the natural solidification of the material. Means that if there is no medium to transfer the heat and the storage tank kept empty and is well insulated. The temperatures have noted to know how long the material will retain in its melting temperature range. The rate of change in the temperature was uniform for all the three materials and after $8 \mathrm{~h}$ the PCMs have come to $45^{\circ} \mathrm{C}$.

\section{DISCUSSIONS}

The melting curve for the two base materials is nearly same. For the eutectic mixture it

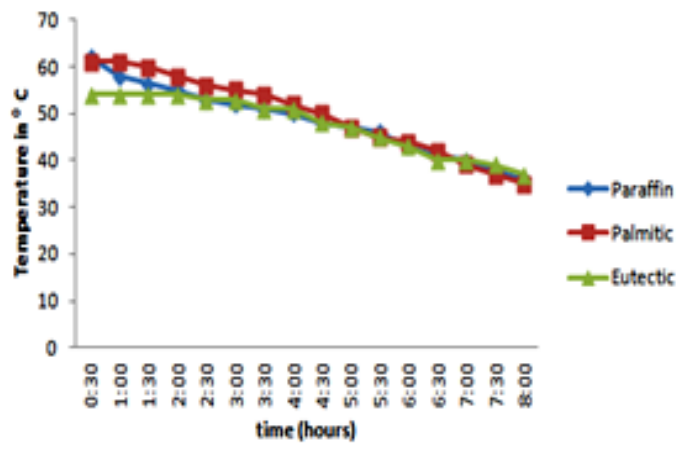

Fig. 6. Natural solidification curve

gradually increased and almost uniform after $50{ }^{\circ} \mathrm{C}$. The decrease in the HTF temperature is proportional to the increase in the PCMs. All the three materials shown nearly same raise of temperatures during the melting process.

In Cold water solidification process the base materials have given their energy within 60 minutes and the raise in cold water is $5^{\circ} \mathrm{C}$. The discharging process was gradual in eutectic mixture and it took $120 \mathrm{~min}$. to give up the $8^{\circ} \mathrm{C}$.

In Hot water solidification process all the three materials possess the same nature. The change in temperature is gradual and it took $7 \mathrm{~h}$ to come to $45^{\circ} \mathrm{C}$.

In natural solidification process the change in temperatures were very slow. In case of the eutectic mixture there were not many changes. After $8 \mathrm{~h}$ the temperature of the material comes down to $45^{\circ} \mathrm{C}$.

Considering the three cases, the natural solidification process is suitable for heating of buildings. The Hot water solidification is suitable for retaining the storage tank temperatures high for an extra of 5-6 hours. Cold water solidification indicates that eutectic mixture take longer time than base materials and able to regain better temperatures than the base materials.

\section{CONCLUSIONS}

The present study, concentrated on the melting time and temperature of the eutectic mixture 
of paraffin wax and palmitic acid (40:60 wt \%) and the compared with the base materials. Spherical balls have used as storage unit for the TES. The discharging process considered in three ways as i) hot water retained in the storage tank, ii) Cold water to raise its temperature and iii) natural solidification by keeping the storage tank empty. Among the three ways natural solidification took $8 \mathrm{~h}$ to come to $45{ }^{\circ} \mathrm{C}$. In hot water solidification process the hot water was able to maintain its higher temperatures for nearly 7 hours. The base materials have solidified with in an hour by transferred $5{ }^{\circ} \mathrm{C}$ temperature to cold water. The eutectic mixture has taken $2 \mathrm{~h}$ time to raise the cold water temperature to $8{ }^{\circ} \mathrm{C}$.

\section{REFERENCES}

1. Jose Perceira, da Cunha. Philip Eames. Applied Energy., 2016, 177, 227-238.

2. Anant Shukla. Buddhi. D. Sawhney R.L.. Renewable and Sustainable Energy Reviews., 2009, 13, 2119-2125.

3. Atul Sharma. Tyagi, V.V. Chen, C.R. Buddhi, D. Renewable and Sustainable Energy Reviews, 2009, 13, 318-345.

4. Saeid Seddegh, Xiaolin Wang Alan D, Henderson. Ziwen Xing. Renewable and Sustainable Energy Reviews, 2015, 49, 517-533.

5. Tian Y. Zhao, C.Y. Applied Energy, 2013, 104, 538-553.

6. Guruprasad Alva, Lingkun Liu. Xiang Huang. Guiyin Fang. Renewable and Sustainable Energy Reviews, 2017, 68, 693-706.

7. Dan Nchelatebe Nkwetta, Fariborz Haghighat. Sustainable Cities and Society, 2014, 10, 87-100.

8. Ruben Baetens, Bjorn Petter Jelle, Arild Gustavsen. Energy and Buildings, 2010, 42, 1361-1368.

9. Yanping Yuan, Nan Zhang, Wenquan Tao, Xiaoling Cao, Yaling HE. Renewable and Sustainable energy Reviews, 2014, 29, 482-498.

10. Antony Gil, Eduard Oro, Albert Castell, Luisa Cabeza F. Applied Thermal Engineering, 2013, 54, 521-527.

11. Heike Schreiber, Franz Lanzerath, Christiane Reinert, Christoph Gruntgens, Andre Bardow. Applied Thermal Engineerin., 2016, 106, 981-991.
12. Esapour M, Hosseini M.J, Ranjbar A.A, Pahamli Y, Bahrampoury R. Renewable Energy, 2016, 85, 1017-1025.

13. Meysam Faegh, Mohammad Behshad Shafli. Desalination, 2017, 409, 128-135.

14. Kevin Merlin, Didier Delaunay, Jerome Soto, Luc Traonvouez. Applied Energy, 2016, 166, 107-116.

15. Medrano M, Yilmaz M.O, Nogues M, Martorell I, Joan Roca, Luisa, Cabeza F. Applied Energy, 2009, 86, 2047-2055.

16. Hosseini M.J, Rahimi M, Bhrampoury R Mechanical Sciences, 2015, 6, 221-234.

17. Mohammad Mehrali.; Sara Tahan Latibari.; Mehdi Mehrali.; Teuu Meurah Indra Mahila.; Hendrik Simon Cornelis Metselaar.; Moammad Sajad naghavi.; Emad Sadeghinezhad.; Amir Reza Akhiani. Applied Thermal Engineering, 2013, 61, 633-640.

18. Felix Regin A, Solanki S.C, Saini J.S. Renewable Energy, 2009, 34, 1765-1773.

19. Jesumathy S.P, Udayakumar M, Sures S. Journal of Mechanical Science and Technology, 2012, 26, 959-965.

20. Bhagyalakshmi Pasam, Rajan K, Senthil Kumar K.R. Applied Mechanics and Materials, 2015, 787 77-81.

21. Mohamed Lachheb, Mustapha Karkri, Fethi Albouchi, Sassi Ben Nasrallah, Magali Fois, Patrik Sobolciak. Composites:Part B, 2014, 66, 518-525. 